\title{
Les doses d'irradiation médicale diagnostique à la population
}

\section{Isabelle Gremion ${ }^{a}$, Beat Stoll ${ }^{b}$ \\ a Haute Ecole de Santé Vaud (HESAV), Lausanne \\ b Institut de santé globale, Université de Genève}

Cet article est un extrait du mémoire de master en santé publique, rédigé par I. Gremion à l'ISG de l'Université de Genève.

Correspondance:

Isabelle Gremion, MPH

Haute Ecole de Santé Vaud

Av. de Beaumont 21

CH-1011 Lausanne

isabelle.gremion[at]hesav.ch

\section{La composition du rayonnement ionisant auquel nous sommes exposés}

Nous sommes en permanence exposés à des rayonnements ionisants, comme les rayonnements cosmiques de l'espace, les rayonnements telluriques dus aux éléments radioactifs de la croûte terrestre et les radionucléides naturels, comme le potassium 40 , qui sont incorporés dans notre organisme. Ce rayonnement est d'origine naturelle et son intensité n'est pas partout la même.

Nous sommes également exposés à de faibles doses de rayonnement artificiel qui proviennent de retombées radioactives d'armes nucléaires testées par le passé, des émissions d'exploitation normale des centrales nucléaires et surtout des applications médicales avec les rayons X. La découverte de ces rayonnements ne date que d'un peu plus d'un siècle et la reconnaissance de leur danger suivit de peu le début de leur utilisation en médecine. Ceci donna jour à la nécessité d'une protection, amenant à la création d'organismes de radioprotection énonçant des concepts pour l'homme, sans pénaliser les pratiques diagnostiques bénéfiques. Les rayonnements dus aux applications médicales représentent la quasi-totalité (98\%) de l'exposition de la population aux irradiations d'origine artificielle.

En Suisse, l'exposition moyenne totale de l'homme aux rayonnements ionisants est évaluée à 5,6 millisievert (mSv) par année (Rapport Radon 2009 de l'Office Fédéral de la Santé Publique, OFSP). La dose associée à des irradiations médicales diagnostiques représente environ un quart de cette dose totale (1,2 mSv).

L'évaluation de la dose résultant de l'utilisation médicale diagnostique des radiations est établie grâce à des enquêtes nationales périodiques, tous les dix ans, effectuées par l'OFSP.

Ces enquêtes, recommandées pour suivre les tendances d'exposition, et dont une compilation synthétisée est régulièrement publiée par le Comité scientifique des Nations Unies pour l'étude des effets des rayonnements ionisants, permettent de déterminer la fréquence des examens et les doses délivrées à une population donnée par la radiologie médicale. Les valeurs indiquées ne représentent qu'une estimation de la dose effective moyenne.

La dernière enquête nationale, menée en 2008 par l'OFSP [1], a montré que la fréquence moyenne des examens était de 1,7 examen par habitant et que la dose effective moyenne par habitant (E) représentait 1,2 mSv. Cette dose moyenne annuelle par habitant a augmenté de 20\% entre 1998 et 2008 .

\section{Zusammenfassung}

Wir sind ständig ionisierenden Strahlen ausgesetzt, solchen natürlichen Ursprungs und solchen künstlicher Natur. Unter den künstlichen Strahlungen stehen jene der medizinischen Strahlendiagnostik an erster Stelle, insbesondere die Röntgenstrahlen im Zusammenhang mit medizinischen Untersuchungen.

Das BAG erfasst landesweit alle zehn Jahre die Daten, die die Häufigkeit von Röntgenuntersuchungen und deren Strahlenexposition evaluieren. Die letzte Erhebung (2008) ergibt einen jährlichen Mittelwert der Strahlenexposition von 1,2 mSv pro Einwohner. Ist dieser steigende Mittelwert den verschreibenden Ärzten bekannt? Und was bedeutet er? Kann mit inm ein Strahlenbelastungsrisiko assoziiert werden?

Les radiographies conventionnelles représentent la plus grande partie des examens réalisés (46\%), suivies des radiographies dentaires $(42 \%)$ et des examens de tomodensitométrie ou CT (6\%).

Bien que ne représentant que $6 \%$ du nombre d'examens, la tomodensitométrie est responsable des $68 \%$ de la dose effective collective.

Les radiographies dentaires, bien qu'importantes au niveau de la fréquence des examens ne représentent quant à elles que moins de $1 \%$ de la dose effective collective.

\section{Que savent les médecins prescripteurs d'imagerie radiologique?}

Si les enquêtes nationales visent à déterminer la fréquence des examens et les doses moyennes délivrées à la population par la radiologie médicale, leurs résultats sont-ils bien connus des médecins prescripteurs d'examens radiologiques? Ces médecins sontils bien informés sur les examens qui délivrent les doses les plus élevées et sur le risque radiologique et en tiennent-ils compte dans leur manière de prescrire les examens radiologiques diagnostiques? Diverses études en Europe montrent que les médecins prescripteurs sous-estiment les doses et les risques liés aux examens radiologiques et n'ont que très peu de connaissances sur le sujet $[2,3,4]$. 
Figure 1

Répartition des examens radiologiques en 2008 en Suisse.

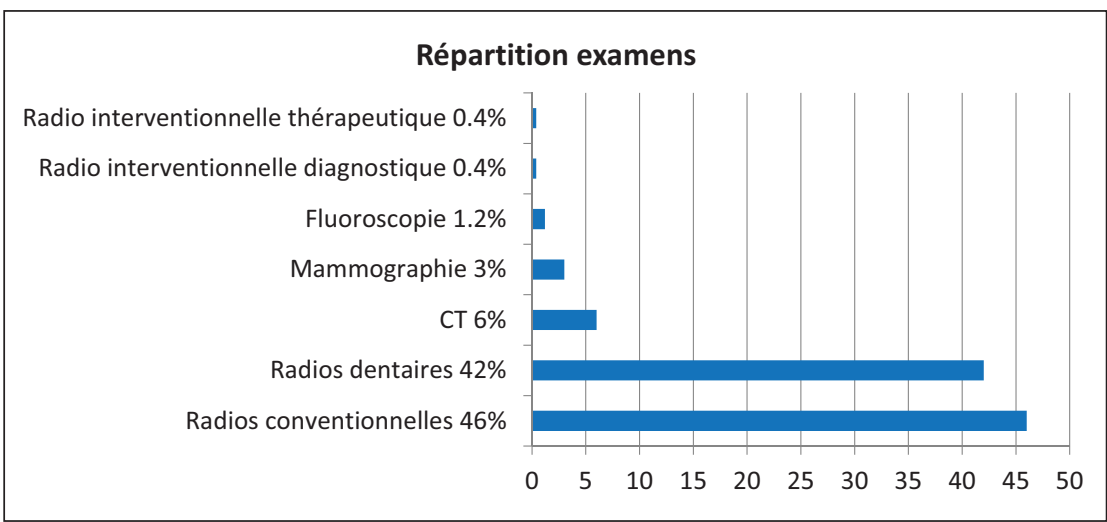

Figure 2

Répartition de la dose annuelle collective par examen radiologique en Suisse en 2008.

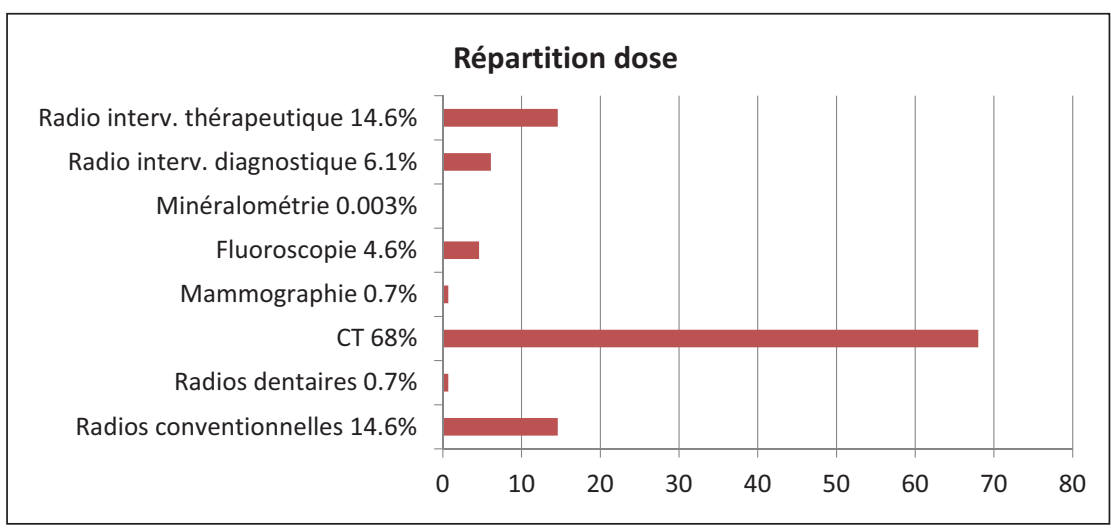

$\mathrm{Au}$ cours de l'élaboration d'un mémoire, nous avons eu l'opportunité de consulter une centaine de médecins pratiquant en Suisse romande (dont un quart dispose d'une qualification pour les examens radiologiques à hautes doses) au travers d'un questionnaire exploratoire en ligne, relatif aux résultats de la dernière enquête nationale, à savoir:

- quelle est la fraction due aux examens radiologiques sur la dose moyenne annuelle totale?

- quels sont les examens qui délivrent la majeure partie de la dose effective moyenne?

- quels sont les examens radiologiques les plus fréquemment réalisés en Suisse?

De plus, deux questions supplémentaires cherchaient à savoir si les médecins se sentent suffisamment informés et à l'aise pour expliquer à leurs patients ce qu'est le risque radiologique et s'ils étaient intéressés à disposer d'informations/recommandations de radioprotection sous forme d'une application pour smartphones et/ou tablettes.

Les médecins connaissent les examens les plus irradiants (CT) et les modalités les plus fréquentes. La grande majorité des médecins consultés sont préoccupés par les doses que délivrent les examens radiologiques. Par contre, ils sous-estiment l'augmentation de la dose effective moyenne à la population entre les deux enquêtes nationales (de 1 à $1,2 \mathrm{mSv}$, soit 20\%). Beaucoup se disent très mal informés sur le risque radiologique et également mal à l'aise pour expliquer ce risque radiologique à leurs patients.

Ils souhaiteraient des informations/recommandations de radioprotection sous forme d'application pour smartphones ou tablettes.

\section{Evaluation et regard critique concernant l'irradiation médicale diagnostique}

Bien que la science n'ait pas apporté de réponses définitives en ce qui concerne le risque radiologique, les connaissances accumulées jusqu'à présent sont cependant suffisantes pour susciter le débat et amener à la réflexion qui consiste à estimer que les rayonnements ionisants sont dangereux, quel que soit le niveau d'exposition, puisque on n'a jamais pu démontrer la non-existence d'effets aux faibles doses.

Avec les examens radiologiques diagnostiques, on reste en principe dans l'ordre de grandeur de doses dites faibles pour lesquelles seuls des effets de type stochastique peuvent apparaître. Cependant, le cumul des doses lié à la répétition des examens peut, pour une petite partie de la population, mener au niveau supérieur des faibles doses (50-100 mSv).

L'évaluation de la dose effective moyenne par habitant en Suisse de 1,2 mSv représente une estimation raisonnable de dose moyenne. Rien ne nous permet d'être certains que cette dose moyenne n'est pas sous-évaluée, car on ne tient pas compte des clichés ratés et répétés, des petits coups de scopie effectués dans bien des salles de radiologie, pour s'assurer du centrage avant de tirer un cliché, ainsi que des séries complémentaires au CT, PET-CT, SPECT-CT.

Cette dose moyenne ne reflète en aucun cas la dose individuelle. Nous savons qu'un grand nombre de personnes n'ont eu aucun examen radiologique en une année, ce qui équivaut à une dose médicale nulle, alors que d'autres doivent subir de nombreux examens radiologiques. Parfois ce sont des enfants qui, par exemple dans des suivis de lymphomes, subissent de nombreux examens CT. Ceci les amène à recevoir des doses de plusieurs dizaines de $\mathrm{mSv}$, avec un risque réel de cancer radio induit des années plus tard.

Pour essayer de quantifier mieux la dose de 1,2 $\mathrm{mSv}$, nous pourrions dire qu'elle correspond, par exemple, à 30 radiographies du thorax chez un adulte.

On peut aussi tenter d'expliquer l'exposition médicale en la comparant à l'exposition naturelle de la population aux radiations ionisantes:

\begin{tabular}{ll} 
Radiographie dentaire & $\begin{array}{l}\text { moins d'un jour } \\
\text { d'irradiation naturelle }\end{array}$ \\
\hline Thorax & 1 semaine d'irradiation naturelle \\
\hline CT cérébral & $\begin{array}{l}\text { près d'un an } \\
\text { d'irradiation naturelle }\end{array}$ \\
\hline CT thoraco-abdominal & $\begin{array}{l}\text { entre } 3 \text { et } 7 \text { ans } \\
\text { d'irradiation naturelle }\end{array}$
\end{tabular}


Les rayons $\mathrm{X}$ constituent un puissant outil diagnostique et sont indissociables de la médecine d'aujourd'hui. Cependant, à ce bénéfice diagnostique est associé un risque radiologique induit par les rayonnements ionisants.

\section{La dose moyenne ne reflète en aucun cas la dose individuelle.}

Les sociétés scientifiques nationales et internationales de radioprotection, parfois en collaboration avec l'OMS, définissent un standard qui exige que les examens radiologiques ne soient effectués que sur la base d'indications cliniques précises et que la dose appliquée soit la plus faible possible (principes de radioprotection de justification et optimisation), pour permettre de répondre à la question clinique posée.

Malgré cela, on observe, au cours de la dernière décennie, une augmentation des examens radiologiques, en particulier des examens CT. Cette augmentation s'explique par le fait que la technique des examens CT a fait de grands progrès, est devenue très accessible et constitue un examen standard de l'imagerie diagnostique. Le CT n'est plus seulement utilisé dans le but de confirmer un diagnostic après examen clinique, mais l'est de plus en plus aussi dans le but d'exclure les présences de pathologies sans qu'il y ait eu examen clinique préalable.

Les améliorations continuelles des appareils et l'utilisation récente de techniques utilisant des modalités mixtes d'imagerie médicale comme le PET/ CT sont également responsables d'augmentations de doses délivrées, tout en étant une plus-value indéniable dans la prise en charge des patients.

Ces derniers eux-mêmes sont souvent demandeurs de ce type d'examens «high-tech» et ne sont probablement que peu préoccupés par le risque radiologique médical. Leur perception du risque radiologique s'exprime plus vite lorsqu'il y a un incident de centrale nucléaire et risque de contamination. Les patients ont besoin d'être informés.

\section{Conclusion et perspectives}

Cette consultation auprès d'un échantillon très restreint de médecins praticiens pourra nous orienter dans le sens qu'il sera pertinent de développer de l'information sur le risque radiologique et sur comment l'expliquer à leurs patients. En Suisse comme ailleurs, la formation en la matière est très limitée. Une étude irlandaise publiée dans le British Journal of Radiology [3] met l'accent sur le besoin de prise de conscience des doses d'irradiation auprès des méde- cins. Une formation ciblée permettrait de connaitre les grandeurs de doses par examen, de reconnaître les examens les plus irradiants et les risques encourus par les patients en fonction des doses auxquelles ils sont exposés.

Nous pensons que les enquêtes nationales de l'OFSP répondent à une partie de la question. Nous suggérons une enquête nationale (avec un questionnaire online) pour mieux cerner ce besoin d'information des médecins.

Etant donné que nous nous trouvons dans une tendance où la dose collective moyenne est en augmentation, il est important d'évaluer régulièrement les doses d'exposition médicale à la population et d'en informer les usagers au sens large du terme. Cependant, si les enquêtes nationales ont leur raison d'être en donnant une photographie de la tendance au niveau de la fréquence des examens et de la dose moyenne annuelle par habitant, elles ne donnent pas satisfaction en ce qui concerne la surveillance individuelle.

Une perspective intéressante pour le futur serait de faciliter le recueil de données et la mise à disposition de toutes les informations de façon la plus automatique possible. Les acteurs de la radioprotection pourraient ainsi disposer d'informations à la fois actualisées et précises, donnant la dose collective, la fréquence des examens, les variations régionales et la répartition parmi les patients.

L'objectif de radioprotection et de réduction de dose en imagerie médicale diagnostique devrait être un enjeu de santé publique faisant intervenir les nombreux acteurs impliqués: médecins, techniciens en radiologie médicale, physiciens médicaux, ingénieurs biomédicaux, patients et autres. Tous à leur niveau doivent pouvoir bénéficier des informations nécessaires, permettant de se questionner sur l'utilisation raisonnable des radiations ionisantes et de favoriser l'émergence d'une culture du risque radiologique.

\section{Références}

1 Aroua A, Samara ET, Bochud FO, Verdun F, Vader RP. Exposure of the Swiss population by Medical X-rays: 2008 Review; 2011.

2 Uri IF. Lack of radiation awareness among referrers: implications and possible solutions. International journal of clinical practice. 2012;66(6):574-81. Epub 2012/5/23.

3 Soye JA, Paterson A. A survey of awareness of radiation dose among health professionals in Northern Ireland. The British journal of radiology. 2008;81(969):725-9. Epub 2008/7/2.

4 Carpeggiani C, Kraft G, Caramella D, Semelka R, Picano E. Radioprotection (un)awareness in cardiologists, and how to improve it. The international journal of cardiovascular imaging. 2012;28(6):1369-74. Epub 2011/08/19. 